\title{
Natural selection influenced the genetic architecture of brain structure, behavioral and
} neuropsychiatric traits

Frank R Wendt ${ }^{1}$, Gita A Pathak ${ }^{1}$, Cassie Overstreet ${ }^{2}$, Daniel S Tylee ${ }^{1}$, Joel Gelernter ${ }^{1,3}$, Elizabeth G Atkinson ${ }^{4}$, Renato Polimanti ${ }^{1, *}$

1. Department of Psychiatry, Yale School of Medicine and VA CT Healthcare System, West Haven, CT 06516, USA

2. National Center for Posttraumatic Stress Disorder, Clinical Neurosciences Division, VA CT Healthcare System and Department of Psychiatry, Yale University School of Medicine

3. Departments of Genetics and Neuroscience, Yale University School of Medicine, New Haven, CT 06510, USA

4. Analytic and Translational Genetics Unit, Massachusetts General Hospital, Boston, MA, USA \& Stanley Center for Psychiatric Research, Broad Institute of MIT and Harvard, Cambridge, MA, USA

*Corresponding author: Renato Polimanti, PhD. Yale University School of Medicine, Department of Psychiatry. VA CT 116A2, 950 Campbell Avenue, West Haven, CT 06516, USA. Phone: +1 (203) 932-5711 x5745. Fax: +1 (203) 937-3897. E-mail: renato.polimanti@yale.edu 


\begin{abstract}
Natural selection has shaped the phenotypic characteristics of human populations. Genome-wide association studies (GWAS) have elucidated contributions of thousands of common variants with small effects on an individual's predisposition to complex traits (polygenicity), as well as wide-spread sharing of risk alleles across traits in the human phenome (pleiotropy). It remains unclear how the pervasive effects of natural selection influence polygenicity in brain-related traits. We investigate these effects by annotating the genome with measures of background (BGS) and positive selection, indications of Neanderthal introgression, measures of functional significance including loss-of-function (LoF) intolerant and genic regions, and genotype networks in 75 brain-related traits. Evidence of natural selection was determined using binary annotations of top $2 \%, 1 \%$, and $0.5 \%$ of selection scores genome-wide. We detected enrichment $(q<0.05)$ of SNP-heritability at loci with elevated BGS ( 7 phenotypes) and in genic (34 phenotypes) and LoF-intolerant regions (67 phenotypes). BGS (top 2\%) significantly predicted effect size variance for trait-associated loci ( $\sigma^{2}$ parameter) in 75 brainrelated traits $\left(\beta=4.39 \times 10^{-5}, p=1.43 \times 10^{-5}\right.$, model $\left.r^{2}=0.548\right)$. By including the number of DSM-5 diagnostic combinations per psychiatric disorder, we substantially improved model fit $\left(\sigma^{2} \sim\right.$ $\mathrm{B}_{\mathrm{Top} 2 \%} \times$ Genic $\times$ diagnostic combinations; model $\left.r^{2}=0.661\right)$. We show that GWAS with larger variance in risk locus effect sizes are collectively predicted by the effects of loci under strong BGS and in regulatory regions of the genome. We further show that diagnostic complexity exacerbates this relationship and perhaps dampens the ability to detect psychiatric risk loci.
\end{abstract}




\section{Introduction}

Genome-wide association studies (GWAS) have identified numerous common genetic

3 variants underlying thousands of human health and disease phenotypes. ${ }^{1}$ Psychiatric,

4 neurological, and mental health related disorders and phenotypes (e.g., personality and social

5 science outcomes) have been investigated by large-scale GWAS, revealing substantial

6 polygenicity, with small effects of loci across the genome contributing additively to a

7 phenotype. ${ }^{2}$ Loci conferring risk or protection also have been shown to have a high degree of

8 pleiotropy, with alleles associated with multiple traits through similar mechanisms. ${ }^{3 ;} 4$ For

9 example, years of education has been negatively genetically correlated with smoking behavior, ${ }^{3}$

10 body mass index, ${ }^{3}$ coronary artery disease, ${ }^{3}$ attention deficit hyperactivity disorder $(A D H D),{ }^{5}$

11 anxiety disorders, ${ }^{5}$ major depressive disorder, ${ }^{5}$ and stroke, ${ }^{5}$ but is positively genetically

12 correlated with high density lipoproteins, ${ }^{3}$ anorexia nervosa,${ }^{5}$ autism spectrum disorder, ${ }^{5}$ bipolar

13 disorder, ${ }^{5}$ obsessive compulsive disorder, ${ }^{5}$ and schizophrenia. ${ }^{5}$ The complex relationship

14 between the shared polygenic architecture of presumably beneficial (e.g., high education) and

15 debilitating (e.g., schizophrenia) traits intensifies a longstanding discussion about the origin and

16 persistence of genetic risk for psychiatric and neurological disorders in the general population.

17 One example of this puzzle is demonstrated with genetic risk for schizophrenia, a disorder

18 observed in approximately $1 \%$ of the general population. ${ }^{6}$ Schizophrenia is often genetically and

19 phenotypically associated with reduced fitness and fecundity. ${ }^{7 ; 8}$ Several hypotheses around this

20 observation suggest that schizophrenia risk loci (1) confer advantage to those unaffected by the

21 disorder or (2) are in strong linkage disequilibrium with positively selected alleles, resulting in

22 an evolutionary trade-off between schizophrenia risk and more beneficial traits (e.g., high

23 intelligence). 7 ; 9-11 
Background selection (BGS), the selective removal of alleles across the genome that

25 confer deleterious effects, has been widely detected in the loci associated with complex traits,

26 including those related to mental health and behavior. ${ }^{12-16}$ The effects of BGS are visible in the

27 contribution of common and rare complex trait risk loci to overall trait heritability estimates:

28 complex trait heritability is generally distributed evenly in common variants across the genome

29 but is nonuniformly distributed across rare variants. ${ }^{13}$ These recent findings suggest that BGS has

30 directly contributed to the degree of polygenicity of complex phenotypes and this relationship

31 appears to be particularly strong in brain-related traits. ${ }^{13 ;}{ }^{16}$ Because of the extremely complex

32 genetic architecture of multifactorial brain-related phenotypes, the sample sizes needed to

33 maximize explainable trait heritability exceeds several million. ${ }^{2}$ Even estimating these required

34 sample sizes is further complicated by the effects of phenotype heterogeneity, which serves to

35 reduce power for the detection of loci in GWAS, and is a systematic feature of diagnosed mental

36 health conditions such as psychiatric disorders. ${ }^{17 ;} 18$

Many phenotypes of interest for large-scale GWAS have specific diagnostic criteria; for

38 psychiatric disorders it is standard practice to refer to the Diagnostic and Statistical Manual of

39 Mental Disorders, $5^{\text {th }}$ Edition (DSM-5). In practice, multi-item symptom check lists based on

40 diagnostic criteria can be employed to classify an individual as a case versus a control. In

41 performing this type of phenotype reduction, underlying differences in symptomology have the

42 potential to produce large degrees of heterogeneity among the individuals being tested. For

43 example, there are 636,120 possible combinations of symptoms which may lead to a

44 posttraumatic stress disorder (PTSD) diagnosis by DSM-5 guidelines. ${ }^{19}$ Similar observations

45 have been made with $A D H D^{20}$ and substance use disorders. ${ }^{21 ; 22}$ While the symptoms used to 
46 define a specific diagnosis are generally highly correlated, these differences may still affect our

47 ability to detect genetic signals associated with phenotype defined by diagnostic criteria.

Since gene discovery can provide information about the disease biology and identify

49 potential treatment targets, it is imperative to study how evolution and diagnostic heterogeneity

50 independently and interactively contribute to the polygenicity of traits related to mental health

51 and disease. The goal of the present study was to investigate how natural selection influences

52 polygenicity across 75 brain-related traits. With respect to psychiatric disorders, we investigated

53 how the addition of heterogeneous trait definitions further contribute to our ability to detect

54 genomic risk loci in psychiatric disorders. We find that both BGS and clinical heterogeneity

55 increase the variance of effect size for risk loci of complex traits, including diagnosed psychiatric

56 disorders, personality traits, internalizing and externalizing behaviors, social science trait

57 outcomes, and brain imaging phenotypes.

\section{Subjects and Methods}

\section{Datasets}

61 GWAS summary association data were accessed from the Psychiatric Genomics

62 Consortium (PGC), Social Science Genetic Association Consortium (SSGAC), UK Biobank

63 (UKB), and UKB Brain Imaging Genetics (BIG) Consortium. All datasets were formatted as

64 standard input for Linkage Disequilibrium Score Regression (LDSC). Observed-scale SNP-based

65 heritability $\left(h^{2}\right)$ was calculated for all traits. For case/control phenotypes, $h^{2}$ was calculated using

66 effective sample sizes and converted to liability scale using population and sample prevalences

67 reported in the respective publications (Table 1). The 1000 Genomes Project Europeans ${ }^{23}$ were 
68 used as the LD reference panel. Traits were selected for partitioning (see Partitioned

69 Heritability) based on $h^{2} \mathrm{z}$-score $\geq 7$ (Table S1) as previously recommended. ${ }^{24}$

$71 \quad$ Partitioned Heritability

$72 \quad$ Heritability partitioning was performed with LDSC using 53 baseline genomic

73 annotations from Finucane, et al. ${ }^{24}$ characterizing important molecular properties such as allele

74 frequency distributions, conserved regions of the genome, and regulatory elements. We created

75 additional genome-wide annotations for genic, loss-of-function (LoF) intolerant, positively

76 selected, negatively selected, and Neanderthal-introgressed positions. These evolutionary

77 annotations are described below using per-SNP measurements obtained directly from the original

78 publications. In line with previous studies, ${ }^{14 ; 25 ; 26}$ annotations of evolutionary pressures were

79 analyzed as bins of the top $2 \%$, top $1 \%$, and top $0.5 \%$ of scores genome-wide. Enrichments were

80 interpreted as follows: (1) all fold-enrichments below zero were considered invalid and not used

81 for any analyses, (2) fold-enrichment values between zero and one indicated $h^{2}$ depletion

82 attributed to an annotation, and (3) fold-enrichments greater than one indicated $h^{2}$ enrichment

83 attributed to an annotation.

84 The genic annotation of the genome was defined by mapping SNPs to genic regions

85 according to the Exome Aggregation Consortium (ExAC) database. ${ }^{27}$ To annotate LoF intolerant

86 regions of the genome, we assigned a probability of loss-of-function (pLI) score to each gene

87 based on the ExAC database. We annotated any SNP in a gene with $\mathrm{pLI} \geq 0.9$ as LoF

88 intolerant. ${ }^{14 ; 27}$ Both annotations were coded in a binary manner: genic versus not-genic and LoF

89 intolerant versus LoF tolerant. 
One measure of BGS was tested. The $B$-statistic describes reduction in local allele

91 diversity as a consequence of purifying selection. This measurement leverages phylogenetic

92 information from other closely related species (e.g., gorilla, chimpanzee, orangutan, and rhesus

93 macaque) to test for evidence of pressures selectively removing alleles at a locus from the human

94 genome. ${ }^{25 ; 28}$ Note that $B$ values were transformed $(1-B)$ such that $B$ near one indicated strong

95 effects of BGS.

96 We tested $h^{2}$ enrichment based on three measures of positive selection. Firstly, we

97 examined two estimates of extended haplotype homozygosity (EHH), which quantifies the

98 number of unique haplotypes that exist in a population given a genetic distance. A high EHH

99 score suggests few haplotypes exist in a population, which occurs when an allele is positively

100 selected, dragging along its linked genetic regions. The integrated haplotype score (iHS)

101 incorporates LD and the ancestral versus derived context of an allele into EHH. Extreme iHS

102 scores signify a difference between individuals with the ancestral and derived versions of the

103 allele. This test probes for ancient selection trends in the context of selection on a particular

104 genotype at a locus. The cross-population extended haplotype homozygosity (XP-EHH) measure

105 compares haplotypes across populations (here we used 1000 Genomes Project African and

106 European super populations), ${ }^{29}$ rather than across ancestral and derived groupings, to detect

107 instances where one allele has reached fixation in one population but remains polymorphic in the

108 other population. XP-EHH provides evidence of more recent positive selection in the time since

109 the two selected populations diverged. ${ }^{29}$ Finally, the composite of multiple signals (CMS) uses a

110 combination of three measures of selection (long haplotypes, differentiated alleles, and high

111 frequency derived alleles) to detect selective events occurring approximately 20-35 thousand

112 years ago..$^{30 ; 31}$ 
114 is quantified by comparing Neanderthal and contemporary human genomes for human genomic

115 positions with high likelihood of originating via admixture events with Neanderthals since the

116 time that these populations diverged. Enrichment of loci with Neanderthal LA in human complex

117 traits would indicate significant contribution of Neanderthal ancestry to those phenotypes. ${ }^{32 ; 33}$

118 Annotations also were generated for summary data describing genotype networks (i.e., a

119 collection of genotypes and their relatedness) across the genome. ${ }^{34 ; 35}$ The attributes of genotype

120 networks used here are: the number of vertices, average path length, number of components, and

121 the average degree of genotypes or network nodes. The number of vertices and average path

122 length of a genotype network describe how a network extends through genotype space and how

123 heterogeneous or genetically distant the genotypes in a population are. Specifically, the number

124 of vertices is equivalent to the number of distinct genotypes in a population. The average path

125 length describes the average of all possible shortest paths between pairs of genotypes within a

126 network. Average path lengths therefore correspond to the number of SNP changes required to

127 move from one node to another. The remaining properties of genotype networks (number of

128 components and average degree of genotypes) have been used to describe the robustness of a

129 locus to mutational events. The number of components in a genotype network is the number of

130 subgroups in a network connected through a continuous path of edges connecting network nodes.

131 Lastly, the average degree of a network is the average degree of all nodes within that network

132 such that nodes without edges are considered "isolates" and have degree zero. All nodes,

133 including degree zero nodes, are included in the calculation of average degree per genotype

134 network. Genotype networks can be studied to identify the sensitivity and survivability of a

135 phenotype given cycles of neutral mutational events. ${ }^{36}$ That is, populations with similar 
136 phenotypes accumulated neutral or slightly deleterious mutations followed by a beneficial

137 mutation that increases population fitness. This type of event fosters development of new

138 genotypes which lie along a similar genotype network (described by the features detailed above).

139 Enrichment of genotype networks may demonstrate the ability of a phenotype to tolerate a

140 population's exploration of genotype space over time and the chance that it tolerates neutral

141 and/or deleterious events in favor of future beneficial evolutionary events. ${ }^{36}$

144 GWAS have detected tens-to-hundreds of loci associated with complex traits but

145 individual locus effect sizes are relatively small. Studying these loci in combination explains

146 substantially greater trait heritability than any individual locus. Descriptive statistics of the effect

147 size distribution for loci associated with complex traits were the main dependent variable of

148 interest in this study. We evaluate how evolutionary pressures (described above) contribute to the

149 descriptive statistics of trait associated loci effect size distribution described by Zhang, et al. ${ }^{2}$

151 package was used to determine effect size distribution descriptive statistics for brain-related

152 traits. ${ }^{2}$ All GWAS for brain-related traits were filtered to include only Hapmap3 SNPs. ${ }^{37}$ As per

153 developer guidelines, ${ }^{2}$ SNPs were removed if (1) their effective sample sizes were less than 0.67

154 times the $90^{\text {th }}$ percentile of the per-SNP sample size distribution, (2) they fell within the major

155 histocompatibility region (these were removed due to the complex LD structure of this region),

156 and (3) they had extremely large effect sizes (per-SNP effect $z$-scores $>80$ ). Descriptive statistics

157 of effect size distribution are defined as follows: (1) $\pi_{\mathrm{c}}$ is the proportion of susceptibility SNPs

158 per trait, (2) $\sigma^{2}$ is the variance parameter for non-null SNPs, and (3) $a$ is the parameter describing 
159 all residual effects not captured by the variance of effect-sizes (e.g., population stratification,

160 underestimated effects of extremely small effect size SNPs, and/or genomic deflation). ${ }^{2}$ Zhang,

161 et $a .^{2}$ compared two- and three-component models of effect size distribution assuming $99 \%$ of

162 SNPs in complex trait GWAS are null and the effect sizes for the remaining $1 \%$ of non-null

163 SNPs follow a normal distribution centered around zero (two-component) or a mixture normal

164 distribution (three-component). Results from Zhang, et al. ${ }^{2}$ support the use of two-component

165 models for autism spectrum disorder, bipolar disorder, major depressive disorder,

166 schizophrenia, college completion, neuroticism, cognitive performance, and intelligence

167 quotient. We therefore considered only the two-component model to calculate effect size

168 distribution descriptive statistics for the brain-related phenotypes analyzed in this study.

171 Many psychiatric disorder diagnoses rely on the number of diagnostic criteria per patient

172 exceeding some threshold. Heterogeneity arises when individuals with the same diagnosis

173 exhibit different combinations of diagnostic criteria. Here we used the psychiatric disorder

174 heterogeneity features described by Olbert, et al. ${ }^{20}$ to evaluate the impact of psychiatric disorder

175 diagnostic heterogeneity on effect size distribution descriptive statistics for GWAS of psychiatric

176 disorders. We focus our analyses on psychiatric disorder total symptoms and diagnostic

177 combinations. Total symptoms are the number of criteria considered for diagnosis according to

178 DSM-5. Diagnostic combinations were defined as any set of symptoms that could meet

179 diagnostic criteria for a disorder dependent upon the presence of a certain combination of

180 symptoms. Based on DSM-5 descriptions of these measures, obsessive compulsive disorder and

181 Tourette syndrome ${ }^{38}$ would be characterized by the same total symptoms and diagnostic 
combinations, so only Tourette syndrome was included in regression analyses because it has a

183 higher $h^{2} z$-score than obsessive compulsive disorder.

Non-Parametric Correlation and Regression Tests

191 measures of evolutionary pressure and observed-scale $h^{2}$ and unstandardized measures of effect

192 size distribution, and (iii) unstandardized measures of all evolutionary pressures, observed-scale

$193 h^{2}$, and measures of effect size distribution. Unless otherwise noted, we discuss results using

194 standardized independent and dependent variables but provide all correlations in the

195 corresponding supplementary information. Non-parametric regression tests included median-

196 based linear regression (MBLM; y x), local regression (locally-estimated scatterplot smoothing

197 or LOESS: $\mathrm{y} \sim \mathrm{x}_{1}+\mathrm{x}_{2}+\mathrm{x}_{\mathrm{n}}$ ), and generalized additive models (GAM: $\mathrm{y} \sim \mathrm{x}_{1}+\mathrm{x}_{2}+\mathrm{x}_{\mathrm{n}}$ and $\mathrm{y} \sim \mathrm{x}_{1}$

$\left.198 \times \mathrm{x}_{2} \times \mathrm{x}_{\mathrm{n}}\right)$ to evaluate the predictive capabilities of linear and additive models on an outcome

199 variable of interest. MBLM computes all lines between each pair of data points and reports the

200 median of the slopes of these lines. Model $r^{2}$ values are not reported due to the median-based

201 nature of the test; however, MBLM slopes and $p$-values for each slope are reported. GAMs were

202 evaluated using nominally significant MBLM predictors. When multiple binary versions of the

203 same genomic annotation were significant predictors of the outcome variable by MBLM (e.g.,

204 enrichment of loci in the top $2 \%, 1 \%$, and $0.5 \%$ of $B$-statistics were all significant predictors of 
outcome Y), the genomic annotation with the lowest $p$-value was included in GAM. GAMs are accompanied by generalized cross-validation (GCV) scores representing mean square error of

207 the model. GCVs associated with GAM estimate the model prediction error without performing

208 formal cross-validation in an out-sample. GCVs closer to zero represent better fitting models.

209 The analysis of variance (ANOVA; anova.gam) feature in the $\mathrm{mgCv}^{39} \mathrm{R}$ package was used to

210 evaluate significant differences in model fit.

Multiple Testing Correction

213 We applied a false discovery rate (FDR) multiple testing correction for the expected

214 proportion of false discoveries among rejected null hypotheses based on the number of tests

215 performed in each analysis. Conversion of test statistic $p$-values to multiple testing corrected $q$ -

216 values was performed in $\mathrm{R}$ using the $\mathrm{p}$. adjust function and the fdr method. ${ }^{40}$ Unless

217 otherwise noted, we report original, unadjusted p-values for enrichment estimates, correlations,

218 and model estimates. For example, anova . gam prints pre-adjusted test statistics; we report

219 these as $q$ instead of $p$.

\section{Results}

222 A total of 75 mental health outcomes were sufficiently heritable ( $h 2 z$-score $\geq 7)$ for

223 partitioning based on evolutionarily relevant functional annotations of the genome (Table S1).

224 These phenotypes were grouped into eleven categories based on general trait similarities: anxiety

225 and related, brain imaging, cognition, cross disorder, depressive and related, eating,

226 externalizing behavior, family adversity, internalizing behavior, neurodevelopmental, and

227 substance use and related (Table S1). 
231 significantly enriched for genic and LoF-intolerant loci, respectively, 34 and 67 of which

232 survived FDR multiple testing correction $(q<0.05$; Figure 1 and Table S1). The top three

233 significant phenotypes were the same for both annotations: schizophrenia, educational

234 attainment, and cognitive performance (Table 1). The greatest $h^{2}$ enrichment of genic loci was

235 observed in the GWAS of T1-weighted left-plus-right caudate volume (enrichment=1.32-fold,

$236 p=0.003$ ). When phenotypes were binned into eleven major categorical domains (Table S1), the

237 brain imaging category demonstrated significantly higher enrichment of $h^{2}$ attributed to genic

238 loci than cognition (difference in mean $=0.174, q=1.47 \times 10^{-5}$ ), depressive and related (difference

239 in mean=0.125, $q=0.002$ ), internalizing behavior (difference in mean=0.130, $q=0.001$ ), and

240 substance use and related (difference in mean $=0.158, q=1.46 \times 10^{-5}$ ) phenotype categories. The $h^{2}$

241 for GWAS of confiding adult relationship (enrichment=2.31-fold, $p=1.16 \times 10^{-4}$ ) and recent easy

242 annoyance (enrichment $=2.31$-fold, $p=1.60 \times 10^{-5}$ ) demonstrated the greatest LoF-intolerance

243 enrichment magnitude. There was no significant difference in enrichment of LoF intolerance loci

244 by phenotype category (ANOVA $p=0.149$ ). Thirty-two phenotypes demonstrated FDR

245 significant enrichment of both genic and LoF intolerant loci $(q<0.05)$; there was a strong overlap

246 between genic and LoF intolerant enrichment magnitudes ( $\rho=0.490, p=0.003$; Figure S1).

247 We next tested whether significant genic and LoF intolerance enrichments were

248 influenced by trait heritability as a proxy representation of effective population size per

249 phenotype. Standardized genic $\left(\rho=0.950, p=2.20 \times 10^{-16}\right)$ and LoF-intolerant $\left(\rho=0.890, p=2.20 \times 10^{-}\right.$

$250{ }^{16}$ ) enrichments were significantly positively correlated with standardized trait $h^{2}$ (Figure S1). 
251 These results are recapitulated in unstandardized correlations where enrichment magnitude for

252 genic and LoF-intolerance annotations was significantly negatively correlated with trait

253 standardized $h^{2} z$-score (standardized $h^{2}$ versus genic enrichment: $\rho=-0.790, p=3.52 \times 10^{-10}$;

254 standardized $h^{2}$ versus LoF intolerance enrichment: $\rho=-0.300, p=0.015$; Figure S1). In other

255 words, GWAS for phenotypes with higher confidence $h^{2}$ estimates tend to show higher

256 confidence enrichment estimates, but these estimates tend to approach 1 (i.e., minimal

257 enrichment). The former observation is expected and suggests that the more accurately trait $h^{2}$ is

258 estimated, the more accurately that $h^{2}$ can be partitioned. ${ }^{24}$ The latter observation is perhaps

259 unexpected and may suggest that the magnitude of $h^{2}$ enrichment attributed to functional

260 annotation is nonuniformly a function of proxy measures of sample size up to a certain level of

261 trait $h^{2}$, visually estimated here to be an approximate $h^{2} z$-score $=15$.

\section{Enrichment of Background Selection}

265 at least one of the genomic annotations created here (i.e., top $2 \%, 1 \%$, and/or $0.5 \%$ of $B$-statistic

266 genome-wide). The only major phenotype domain not demonstrating enrichment of BGS was

267 eating, comprising the anorexia nervosa and recent appetite change phenotypes. Elevated BGS

268 was detected at the level of FDR significance $(q<0.05)$ in the GWAS of seven phenotypes, all of

269 which demonstrated at least nominally significant BGS enrichment at all three genomic

270 annotations and when using the $B$-statistic as a continuous annotation (Table 2). Three of these

271 phenotypes also demonstrated depletion of positive selective pressures at various levels of

272 thresholding each annotation of the genome: (1) the $h^{2}$ of cognitive performance was depleted of

273 SNPs in the top $2 \%$ of CMS scores (0.112-fold depletion, $p=0.002)$ and SNPs in the top $1 \%$ of 
274 iHS scores (0.207-fold depletion, $p=0.012)$, (2) schizophrenia was depleted of SNPs in the top

$2752 \%$ of XP-EHH scores (0.216-fold depletion, $p=0.005)$, and (3) verbal numeric reasoning was

276 depleted for SNPs in the top 2\% of XP-EHH scores (0.190-fold depletion, $p=0.023)$, SNPs in the

277 top $2 \%$ of CMS scores (0.101-fold depletion, $p=0.028)$, and SNPs in the top $2 \%$ of iHS scores

278 (0.371-fold depletion, $p=0.001)$. Table S1 lists enrichment estimates and $p$-values for all three

279 annotations of each selection statistic (BGS, iHS, XP-EHH, and CMS). Because BGS results in

280 reduced genetic variation, these enrichment results for BGS and positive selection may be

281 interpreted as concordant evidence for the strong effect of BGS on complex traits.

282 We next tested whether standardized BGS enrichments were associated with standardized

$283 h^{2}$ (like those observations with genic and LoF intolerant loci). There were significant positive

284 associations between standardized $h^{2}$ and BGS at all three genomic partitions: $h^{2}$ versus

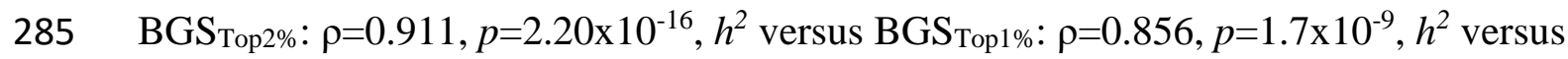

286 BGS $_{\text {Top } 0.5 \%}: \rho=0.751, p=9.81 \times 10^{-6}$. Unstandardized correlations reiterate observations with genic

287 and LoF intolerance correlations where those phenotypes with high confidence $h^{2}$ estimates (e.g.,

$288 h^{2} z$-score greater than $\sim 15$ ) generally showed no relationship between $h^{2}$ and BGS enrichment

289 (Figure S2).

291 Enrichment of Neanderthal Introgression

292 Posterior probability of Neanderthal LA indicates contribution of Neanderthal genomes

293 to a particular phenotype. ${ }^{33 ; 41 ; 42}$ The observed scale $h^{2}$ of one phenotype (UK Biobank

294 neuroticism score) was significantly depleted of SNPs in the top $2 \%$ of Neanderthal LA 0.358 -

295 fold depletion, $p=8.61 \times 10^{-6}$ ). This phenotype also exhibited nominally significant enrichment of

296 SNPs demonstrating evidence of BGS in the top $2 \%$ of $B$-statistic (1.57-fold enrichment, 

$p=0.003)$ and top $0.5 \%$ of $B$-statistic (2.28-fold enrichment, $p=0.002)$. It has been shown that per-SNP measures of Neanderthal LA and $B$-statistic are significantly correlated ${ }^{33}$ however we demonstrate, with the $\tau c$ statistic calculated in LDSC, ${ }^{14 ; 24}$ that enrichment of Neanderthal LA in the UKB phenotype neuroticism score was independent of all other annotations of the genome investigated in this study $\left(p=1.41 \times 10^{-4}\right) .{ }^{41}$

\section{Correlates of Effect Size Distribution}

Parameters describing effect size distribution per phenotype $\left(\pi_{\mathrm{c}}, \sigma^{2}\right.$, and $\left.a\right)$ were generated using the GENESIS R package (Figure 2). ${ }^{2}$ Three effect size distribution outliers were detected with measurements of at least three standard deviations from the mean. First, left plus

307 right caudate volume ( $z$-score $=3.06)$ was an outlier with respect to the $\sigma^{2}$ parameter, highlighting

308 a wide distribution of variant effect sizes underlying this phenotype. Second, anorexia nervosa

$309(z$-score $=3.75)$ and bipolar disorder $(z$-score $=5.78)$ were outliers with respect to the $a$ parameter,

310 suggesting possible residual population stratification underlying these GWAS. Notably, neither

311 phenotype is an outlier with respect to their intercept from LDSC: anorexia nervosa

312 intercept $=1.03 \pm 0.010$ and bipolar disorder intercept $=1.01 \pm 0.008$.

We used the non-parametric Spearman correlation test to evaluate linear relationships

314 between effect size distribution parameters $\left(\pi_{\mathrm{c}}, \sigma^{2}\right.$, and $a$ ) and (1) BGS, (2) genic and LoF

315 intolerance, (3) trait $h^{2}$ (considering liability-scale $h^{2}$ for binary traits), and (4) diagnostic

316 heterogeneity (psychiatric disorders only). The phenotype attributes total symptoms and

317 diagnostic combinations were obtained from Olbert, et al. 2014 and describe how the total

318 number of DSM-5 criteria per psychiatric disorder contributes to diagnostic heterogeneity. ${ }^{20}$

319 There were no statistically significant differences in correlation when outliers were removed so 
320 all reported correlations included the complete sample. Standardized genic and LoF-intolerance

321 enrichments significantly predicted all three effect size parameters $\left(\rho_{\pi \mathrm{c} \_ \text {genic }}=0.851, p=2.20 \mathrm{x} 10^{-16}\right.$;

$322 \rho_{\pi \mathrm{c} \_ \text {LoF }}=0.600, p=4.68 \times 10^{-7} ; \rho_{\sigma 2 \_ \text {genic }}=0.862, p=2.20 \times 10^{-16} ; \rho_{\sigma 2 \_ \text {LoF }}=0.599, p=5.07 \times 10^{-7} ;$

$323 \rho_{a \_ \text {genic }}=0.798, p=5.72 \times 10^{-9} ; \rho_{a \_ \text {LoF }}=0.736, p=2.20 \times 10^{-16}$; Figure 3$)$. Using unstandardized

324 measures of genic and LoF intolerance enrichment, we observed no relationship between LoF

325 intolerance and effect size distribution; however, enrichment of genic loci was negatively

326 correlated with $\pi_{\mathrm{c}}\left(\rho_{\pi \mathrm{c} \_ \text {genic }}=-0.682, p=1.52 \times 10^{-6}\right)$ and positively correlated with $\sigma^{2}\left(\rho_{\sigma 2}\right.$

327 genic $\left.=0.546, p=2.33 \times 10^{-4}\right)$. This suggests that $h^{2}$ enrichment attributed to loci in genic regions of

328 the genome (1) decreases the number of detectable risk loci and (2) increases the variance

329 associated with effect size estimates for those risk loci that can be detected.

330 Using only phenotypes with at least nominal enrichment of BGS, all three standardized

331 effect size distribution parameters were nominally correlated with standardized BGS: $\pi_{\mathrm{c}}$

$332\left(\rho_{\pi \mathrm{c} \_ \text {Btop } 2 \%}=0.557, \mathrm{p}=3.59 \times 10^{-7} ; \rho_{\pi \mathrm{c} \_ \text {Btop } 1 \%}=0.557, p=5.49 \times 10^{-7} ; \rho_{\pi \mathrm{c} \_ \text {Btop } 0.5 \%}=0.575, p=1.26 \times 10^{-7}\right)$,

$333 \sigma^{2}\left(\rho_{\sigma 2}{ }_{-}\right.$Btop $2 \%=0.514, p=3.39 \times 10^{-6} ; \rho_{\sigma 2}{ }_{-}$Btop $1 \%=0.549, p=5.16 \times 10^{-7} ; \rho_{\sigma 2}{ }_{-}$Btop $0.5 \%=0.613, p=9.80 \times 10^{-}$

$\left.334{ }^{9}\right)$, and $a\left(\rho_{a_{-} \text {Btop } 2 \%}=0.688, p=2.20 \times 10^{-16} ; \rho_{a_{-} \text {Btop } 1 \%}=0.632, p=9.18 \times 10^{-10} ; \rho_{a_{-}}\right.$Btop0.5\% $=0.543$,

$335 p=7.49 \times 10^{-7}$; Figure 3). Unstandardized analyses reveal that higher enrichment of BGS generally

336 associated with reduced proportion of risk loci $\left(\pi_{\mathrm{c}} ; \rho_{\pi c_{-} B t o p 2 \%}=-0.493, p=0.002 ; \rho_{\pi c_{-} B t o p 1 \%}=-0.462\right.$,

$337 p=0.012)$, increased variance among those non-null risk loci $\left(\sigma^{2} ; \rho_{\sigma 2}{ }_{-}\right.$Btop2\% $\left.=0.413, p=0.009\right)$, and

338 decreased residual effects (e.g., population stratification, underestimated effects of extremely

339 small effect size SNPs, and/or genomic deflation) as measured by the $a$ parameter $\left(\rho_{a_{-}}\right.$Btop $0.5 \%=-$

$\left.3400.642, p=5.50 \times 10^{-4}\right)$. These results highlight the relative importance of BGS on detection of

341 genetic risk for complex phenotypes. Considering psychiatric diagnoses only, there were no 
342 detectable correlative relationships between measures of heterogeneity (total symptoms and

343 diagnostic combinations) and effect size distribution $\left(\pi_{\mathrm{c}}, \sigma^{2}\right.$, and $\left.a\right)$.

345 Predicting Effect Size Distributions

We next tested the ability of each unstandardized trait property (BGS, genic and LoF

347 intolerance enrichment, and heterogeneity features) to predict effect size distribution descriptive

348 statistics $\left(\pi_{\mathrm{c}}, \sigma^{2}\right.$, and $\left.a\right)$ using median-based linear models (MBLM) (Figure 4). For this analysis,

349 Spearman correlations were not used to inform MBLM testing because of potential effects of (1)

350 confounder bias, (2) collider bias, and/or (3) incidental cancellation potentially resulting in (a) a

351 correlated independent variable not predicting an effect size distribution descriptive statistic or

352 (b) an uncorrelated independent variable predicting an effect size distribution descriptive

353 statistic. Therefore, enrichment of genic, LoF intolerant, and all partitions of BGS were used in

354 MBLM to predict $\pi_{\mathrm{c}}, \sigma^{2}$, and $a$ regardless of their pairwise correlations. Though highly correlated

355 with effect size distributions (Figures S1 and S2), standardized and unstandardized trait $h^{2}$ was

356 not used to predict effect size distribution parameters because of its representation of effective

357 population size in case-control phenotypes which varies considerably across phenotypes.

After multiple testing correction, the $\pi_{\mathrm{c}}$ parameter (i.e., proportion of susceptibility loci)

359 was predicted by enrichment of genic, LoF intolerant, and all three BGS partitions (Figure 4).

360 After multiple testing correction, the $\sigma^{2}$ parameter (i.e., the variance parameter for non-null

361 SNPs) was predicted independently by enrichment of all three BGS partitions and genic loci. In

362 the additive model of $\sigma^{2}$, enrichment of $h^{2}$ attributed to loci in the top $2 \%$ of genome-wide $B$ -

363 statistic was the only significant predictor $\left(\beta=4.39 \times 10^{-4}, p=1.43 \times 10^{-5}\right.$; full model $\left.r^{2}=0.548\right)$. In

364 the absence of out-sample cross-validation, GCV estimates associated with model fit $\left(r^{2}\right)$ 
represent a prediction error estimate whereby smaller GCV values indicate greater model fit. For

366 the additive model of $\sigma^{2}$, we observed a very small prediction error in the model fit,

$367 \mathrm{GCV}=5.79 \times 10^{-10}$. Interactive models of $\pi_{\mathrm{c}}$ and $\sigma^{2}$ generally explained comparable variances in

368 the outcome variable of interest but lack significant individual or interactive predictors: $\pi_{\mathrm{c}} \sim$

$369 \mathrm{~B}_{\mathrm{Top} 2 \%} \times$ genic $\times$ LoF full model $r^{2}=0.347, \mathrm{GCV}=2.72 \times 10^{-5}$ and $\sigma^{2} \sim \mathrm{B}_{\mathrm{Top} 2 \%} \times$ genic full model

$370 r^{2}=0.528, \mathrm{GCV}=4.80 \times 10^{-10}$. The $a$ parameter $($ i.e., residual effects not captured by the variance of

371 effect-sizes) was only predicted by $h^{2}$ enrichment attributed to loci in the top $0.5 \%$ of $B$-statistic

372 scores $\left(\right.$ MBLM $\left.\beta=-1.29 \times 10^{-7}, p=2.48 \times 10^{-5}\right)$ and therefore additive and interactive models were

373 not tested.

374 We next focused on a subset of phenotypes for which diagnostic heterogeneity

375 information exists $\left(\right.$ ADHD $_{2}{ }^{43}$ anorexia nervosa,${ }^{44}$ autism spectrum disorder,${ }^{45}$ bipolar disorder ${ }^{46}$

376 major depressive disorder,${ }^{47}$ schizophrenia, ${ }^{48}$ anxiety, ${ }^{49}$ and Tourette syndrome,${ }^{38}$ Figure 4 and

377 Table S1). With these phenotypes we tested whether diagnostic information such as number of

378 diagnostic combinations and total symptoms contribute to risk locus effect size distribution

379 descriptive statistics. First, we re-evaluated the original additive models (see above) in this

380 subset and recapitulated the relationship between the $\sigma^{2}$ parameter, BGS, and genic loci (model

$\left.381 r^{2}=0.268, \mathrm{GCV}=7.58 \times 10^{-10}\right)$ but did not identify any independent significant predictors of $\sigma^{2}$. The

382 additive model for the effects of BGS, genic, and LoF intolerance enrichments on $\pi_{\mathrm{c}}$ were

383 discordant when applied to psychiatric disorders only (model $\left.r^{2}=-0.126, \mathrm{GCV}=1.89 \times 10^{-5}\right)$ and

384 again no significant individual predictors were identified. Conversely, the interactive models

385 exhibited comparable (or increased) and concordant model fit in the subset of psychiatric

386 disorders $\left(\pi_{\mathrm{c}} r^{2}=0.946, \mathrm{GCV}=1.01 \times 10^{-4}\right.$ and $\left.\sigma^{2} r^{2}=0.513, \mathrm{GCV}=4.80 \times 10^{-10}\right)$. Next, we included

387 measures of phenotype heterogeneity into the additive and interactive models. The only 
$\left.391 p_{\text {difference }}=0.411\right)$ with no significant contributors, suggesting no greater prediction potential than

392 the original additive model. Including the total symptoms measure into the interactive model of

$393 \sigma^{2}$ modestly increased model fit: $\sigma^{2} \sim$ B (Top 2\%) $\times$ Genic $\times$ Total Symptoms; model $r^{2}=0.661$,

$394 \mathrm{GCV}=1.82 \times 10^{-9}\left(p_{\text {difference }}=0.401\right)$.

\section{Discussion}

The effect size distribution of a phenotype may be used to infer the total sample size

398 required for risk locus detection as well as the associated genetic variance explained. ${ }^{2}$ Mental

399 health and disease phenotypes typically demonstrate narrow effect size distributions (i.e., normal

400 distributions with most data points distributed tightly around zero) supporting that they are (1)

401 highly polygenic and (2) the additive result of SNPs conferring small effects. ${ }^{2}$ Here we

402 investigated how these effect sizes and their distribution are shaped by natural selection.

403 Genetic risk for schizophrenia, blood pressure, body mass index, major depressive

404 disorder, and others is influenced by natural selection. ${ }^{13 ; 14 ; 16 ; 50}$ The concept of genome-wide

405 flattening was used to demonstrate that low-frequency variants contribute less to the polygenicity

406 of a trait than common variants, and that negative selection constrains effect sizes for these

407 common variants. ${ }^{13}$ Flattening describes the reduced effect size distributions for complex traits

408 subjected to BGS, resulting in a highly polygenic architecture consisting of many loci with

409 relatively small effects. Using common genetic variation and three separate measures of effect

410 size distributions describing (1) the number of non-null risk loci, (2) the variance of non-null risk 
411 loci effect size estimates, and (3) parameter representing residual variance not attributed to non-

412 null risk loci, we converge on comparable findings and replicate the detection of BGS

413 influencing schizophrenia risk. ${ }^{14}$ We additionally replicate that the genetic risk loci for

414 schizophrenia confer extremely small effects on the phenotype due to the selective removal of

415 larger effect variants from the genome over evolutionary time. Here, we extend this observation

416 to 48 other mental health and disease phenotypes and identify traits whose risk loci exhibit an

417 overrepresentation of BGS. Seven phenotypes (bipolar disorder, cognitive performance, drinks

418 per week, educational attainment, schizophrenia, reaction time, and verbal numeric reasoning)

419 survived multiple testing correction, suggesting a significant influence of BGS on their common

420 genetic variation. We also detect an overabundance of genic and LoF intolerant loci contributing

421 to phenotype $h^{2}$, implying that functionally important regions of the genome contribute the most

422 to $h^{2} .13 ; 14 ; 27 ; 51$

423 Three phenotypes demonstrated enrichment of BGS, genic, and LoF intolerance:

424 cognitive performance, educational attainment, and schizophrenia. These overlapping

425 phenotypes demonstrate negatively genetically correlated and arguably advantageous (cognitive

426 performance and educational attainment) and disadvantageous (schizophrenia) features of the

427 mental health phenome, potentially due to evolutionary tradeoffs associated with similar

428 biological effects. The persistence of risk-conferring (i.e., deleterious) loci in regions of the

429 genome intolerant to mutation and at common frequency may at first be viewed as paradoxical.

430 We and others hypothesize that this paradox could be attributed to BGS, which selectively

431 removes haplotypes from the population that contain large effect deleterious mutations. ${ }^{52 ; 53}$ This

432 in turn reduces genetic diversity and allows for small-effect variants (or haplotypes) to rise in 
433 frequency to the levels of common variation detected by GWAS of the mental health and disease 434 phenome. ${ }^{14 ; 27 ; 52-54}$

435 We next evaluated the ability of BGS, genic, and LoF intolerance enrichments to predict

436 the effect size distributions of the mental health and disease phenome. We identified two models

437 for predicting the variance parameter $\left(\sigma^{2}\right)$ of effect size distributions using BGS and genic locus

438 enrichment: (1) an additive model that explained $54.8 \%$ of the variance in $\sigma^{2}$ and (2) an

439 interactive model that explained $52.8 \%$ of the variance in $\sigma^{2}$. These data explicitly quantify the

440 effects of natural selection on the distribution of effects sizes underlying GWAS of complex

441 traits. However, they do not suggest that BGS acts directly on the analyzed phenotypes. They

442 may instead point to strong effects of BGS on pleiotropic regions of the genome shared by these

443 phenotypes. ${ }^{12}$ Only the interactive model performed modestly well when tested in psychiatric

444 disorders only, explaining $51.3 \%$ of the variance in $\sigma^{2}$. After incorporating phenotype

445 heterogeneity information, $66.1 \%$ of variance in the $\sigma^{2}$ parameter (i.e., the variance in effects

446 sizes for non-null risk loci) for psychiatric disorders was explained by the interaction between

447 BGS, loci in genic regions of the genome, and the total number of symptoms included in

448 psychiatric disorder diagnosis, though this improvement over the model excluding total symptom

449 count was not significant.

450 Though increased BGS and genic locus enrichment produce greater $\sigma^{2}$ estimates (greater

451 effect size variance, implying some relatively large and some relatively small effect risk loci),

452 increased phenotype heterogeneity from total symptom counts may reduce the potential for

453 discovering (1) greater numbers of risk loci and (2) higher risk conferring loci. This is likely

454 especially true for traits like $A D H D$ and $P T S D$ which have substantially greater symptom counts

455 (which can be considered as different phenotypically and of which only a subset are required for 
456 diagnosis) than other psychiatric disorders. ${ }^{20}$ These data also lend support to the trends of (1)

457 utilizing continuous phenotype definitions (e.g., GWAS of PTSD Check List (PCL) criterion

458 count $^{55}$ ) instead of case-control diagnoses, where appropriate, and (2) studying endophenotypes

459 rather than diagnoses (i.e., phenotypes connecting behavioral symptoms or psychopathology

460 with well-understood biological phenotypes; e.g., smooth pursuit eye movements as an

461 intermediate phenotype for schizophrenia). ${ }^{56-58}$

462 Our study has three primary limitations. First, a model predicting the effect size

463 distribution variance parameter $\left(\sigma^{2}\right)$ of psychiatric disorders is inherently limited in the number

464 of observations contributing to the development of said model. The scarcity of data contributing

465 to model training likely drives this model towards overfitting and a lack of generalizability, even

466 via cross-validation. This is a difficult issue to tackle, however, due to (1) the motivation to

467 increase the training set size, (2) decisions of appropriate test set size, and (3) lack of large

468 numbers of psychiatric disorders to test. We do not attempt to cross validate this model due to a

469 lack of data to do so, but we do demonstrate that the original model of interactive effects

470 between genic and BGS loci persisted in psychiatric disorders. Second, two psychiatric disorders

471 (anorexia nervosa and bipolar disorder) were statistical outliers with respect to $a$, which

472 captures residual variance in effect size estimates. Though not evident by their LDSC intercept

473 estimates, it is possible that these analyses harbor residual population stratification requiring

474 longer LD-blocks to resolve. ${ }^{59}$ Finally, we use enrichment values to make predictions about

475 effect size distribution outcomes. The precise magnitude of enrichment may change depending

476 on (1) LDSC model structure ${ }^{12 ; 24 ; 51}$ and, as we demonstrate, (2) robust standardized $h^{2}$ scores.

477 Therefore, this model will require further refinement as GWAS for complex phenotypes continue

478 to grow. 
This study supports the extensive effect of BGS and overrepresentation of genic and LoF-

480 intolerant loci in the GWAS of complex mental health and disease phenotypes. These features

481 were capable of predicting the variance in genetic risk effect sizes for these neuropsychiatric

482 phenotypes. We demonstrate that psychiatric disorder genetic risk effect sizes may be masked by

483 phenotype heterogeneity and elucidate genetic and evolutionary evidence in favor of the

484 continuous and/or intermediate phenotype approaches to studying the genetics of psychiatric

485 disorders and complex phenotypes. These data begin to unravel questions of "how" and "why"

486 human brain-related GWAS suffer a substantial burden from large sample size requirements by

487 demonstrating how the combined effects of functional regions and natural selection influence

488 SNP effect size distributions. This research is supported in part by the Department of Veterans

489 Affairs Office of Academic Affiliations Advanced Fellowship Program in Mental Illness

490 Research and Treatment, the Department of Veterans Affairs National Center for Post-Traumatic

491 Stress Disorder Clinical Neurosciences Division, and the VA Connecticut Healthcare System.

492 The views expressed here are the authors' and do not necessarily represent the views of the

493 Department of Veterans Affairs.

494

495 Supplemental Data

496 Supplemental data include one table and two figures.

498 Acknowledgements

499 This study was supported by the Simons Foundation Autism Research Initiative (SFARI

500 Explorer Award: 534858), the American Foundation for Suicide Prevention (YIG-1-109-16), the

501 National Institutes of Health (R21 DC018098 and R21 DA047527), and the National Center for 
502 PTSD of the U.S. Department of Veterans Affairs. This research is supported in part by the

503 Department of Veterans Affairs Office of Academic Affiliations Advanced Fellowship Program

504 in Mental Illness Research and Treatment, the Department of Veterans Affairs National Center

505 for Post-Traumatic Stress Disorder Clinical Neurosciences Division, and the VA Connecticut

506 Healthcare System. The views expressed here are the authors' and do not necessarily represent

507 the views of the Department of Veterans Affairs.

508

509 Declaration of Interests

510 The authors declare no competing interests.

\section{Web Resources}

513 PGC data download: https://www.med.unc.edu/pgc/download-results/; SSGAC data

514 download: https://www.thessgac.org/data; UKB data showcase:

515 http://biobank.ndph.ox.ac.uk/showcase/; UKB Brain Imaging Genetics server - version 2.0:

516 http://big.stats.ox.ac.uk/ 


\section{References}

1. Buniello, A., MacArthur, J.A.L., Cerezo, M., Harris, L.W., Hayhurst, J., Malangone, C., McMahon, A., Morales, J., Mountjoy, E., Sollis, E., et al. (2019). The NHGRI-EBI GWAS Catalog of published genome-wide association studies, targeted arrays and summary statistics 2019. Nucleic Acids Res 47, D1005-D1012.

2. Zhang, Y., Qi, G., Park, J.H., and Chatterjee, N. (2018). Estimation of complex effect-size distributions using summary-level statistics from genome-wide association studies across 32 complex traits. Nat Genet 50, 1318-1326.

3. Bulik-Sullivan, B., Finucane, H.K., Anttila, V., Gusev, A., Day, F.R., Loh, P.R., Duncan, L., Perry, J.R., Patterson, N., Robinson, E.B., et al. (2015). An atlas of genetic correlations across human diseases and traits. Nat Genet 47, 1236-1241.

4. Bulik-Sullivan, B.K., Loh, P.R., Finucane, H.K., Ripke, S., Yang, J., Patterson, N., Daly, M.J., Price, A.L., and Neale, B.M. (2015). LD Score regression distinguishes confounding from polygenicity in genome-wide association studies. Nat Genet 47, 291-295.

5. Anttila, V., Bulik-Sullivan, B., Finucane, H.K., Walters, R.K., Bras, J., Duncan, L., EscottPrice, V., Falcone, G.J., Gormley, P., Malik, R., et al. (2018). Analysis of shared heritability in common disorders of the brain. Science 360.

6. Sullivan, P.F., Kendler, K.S., and Neale, M.C. (2003). Schizophrenia as a complex trait: evidence from a meta-analysis of twin studies. Arch Gen Psychiatry 60, 1187-1192.

7. Mullins, N., Ingason, A., Porter, H., Euesden, J., Gillett, A., Olafsson, S., Gudbjartsson, D.F., Lewis, C.M., Sigurdsson, E., Saemundsen, E., et al. (2017). Reproductive fitness and genetic risk of psychiatric disorders in the general population. Nat Commun 8, 15833. 
8. Power, R.A., Kyaga, S., Uher, R., MacCabe, J.H., Langstrom, N., Landen, M., McGuffin, P., Lewis, C.M., Lichtenstein, P., and Svensson, A.C. (2013). Fecundity of patients with schizophrenia, autism, bipolar disorder, depression, anorexia nervosa, or substance abuse vs their unaffected siblings. JAMA Psychiatry 70, 22-30.

9. Jarvik, L.F., and Deckard, B.S. (1977). The Odyssean personality. A survival advantage for carriers of genes predisposing to schizophrenia? Neuropsychobiology 3, 179-191.

10. Lawn, R.B., Sallis, H.M., Taylor, A.E., Wootton, R.E., Davey Smith, G., Davies, N.M., Hemani, G., Fraser, A., Penton-Voak, I.S., and Munafo, M.R. (2019). Comment on the Relationship Between Common Variant Schizophrenia Liability and Number of Offspring in the UK Biobank. Am J Psychiatry 176, 573-574.

11. Purcell, S.M., Moran, J.L., Fromer, M., Ruderfer, D., Solovieff, N., Roussos, P., O'Dushlaine, C., Chambert, K., Bergen, S.E., Kahler, A., et al. (2014). A polygenic burden of rare disruptive mutations in schizophrenia. Nature 506, 185-190.

12. Gazal, S., Finucane, H.K., Furlotte, N.A., Loh, P.R., Palamara, P.F., Liu, X., Schoech, A., Bulik-Sullivan, B., Neale, B.M., Gusev, A., et al. (2017). Linkage disequilibriumdependent architecture of human complex traits shows action of negative selection. Nat Genet 49, 1421-1427.

13. O'Connor, L.J., Schoech, A.P., Hormozdiari, F., Gazal, S., Patterson, N., and Price, A.L. (2019). Extreme Polygenicity of Complex Traits Is Explained by Negative Selection. Am J Hum Genet 105, 456-476.

14. Pardinas, A.F., Holmans, P., Pocklington, A.J., Escott-Price, V., Ripke, S., Carrera, N., Legge, S.E., Bishop, S., Cameron, D., Hamshere, M.L., et al. (2018). Common 
schizophrenia alleles are enriched in mutation-intolerant genes and in regions under strong background selection. Nat Genet 50, 381-389.

15. Ward, L.D., and Kellis, M. (2012). Evidence of abundant purifying selection in humans for recently acquired regulatory functions. Science 337, 1675-1678.

16. Zeng, J., de Vlaming, R., Wu, Y., Robinson, M.R., Lloyd-Jones, L.R., Yengo, L., Yap, C.X., Xue, A., Sidorenko, J., McRae, A.F., et al. (2018). Signatures of negative selection in the genetic architecture of human complex traits. Nat Genet 50, 746-753.

17. Kulminski, A.M., Loika, Y., Culminskaya, I., Arbeev, K.G., Ukraintseva, S.V., Stallard, E., and Yashin, A.I. (2016). Explicating heterogeneity of complex traits has strong potential for improving GWAS efficiency. Sci Rep 6, 35390.

18. Manchia, M., Cullis, J., Turecki, G., Rouleau, G.A., Uher, R., and Alda, M. (2013). The impact of phenotypic and genetic heterogeneity on results of genome wide association studies of complex diseases. PLoS One 8, e76295.

19. Galatzer-Levy, I.R., and Bryant, R.A. (2013). 636,120 Ways to Have Posttraumatic Stress Disorder. Perspect Psychol Sci 8, 651-662.

20. Olbert, C.M., Gala, G.J., and Tupler, L.A. (2014). Quantifying heterogeneity attributable to polythetic diagnostic criteria: theoretical framework and empirical application. $\mathrm{J}$ Abnorm Psychol 123, 452-462.

21. Agrawal, A., Edenberg, H.J., and Gelernter, J. (2016). Meta-Analyses of Genome-Wide Association Data Hold New Promise for Addiction Genetics. J Stud Alcohol Drugs 77, 676-680.

22. Polimanti, R., Walters, R.K., Johnson, E.C., McClintick, J.N., Adkins, A.E., Adkins, D.E., Bacanu, S.-A., Bierut, L.J., Bigdeli, T.B., Brown, S., et al. (2019). Leveraging genome- 
wide data to investigate differences between opioid use $<\mathrm{em}>\mathrm{vs}</ \mathrm{em}>$. opioid dependence in 41,176 individuals from the Psychiatric Genomics Consortium. bioRxiv, 765065.

23. Auton, A., Brooks, L.D., Durbin, R.M., Garrison, E.P., Kang, H.M., Korbel, J.O., Marchini, J.L., McCarthy, S., McVean, G.A., and Abecasis, G.R. (2015). A global reference for human genetic variation. Nature 526, 68-74.

24. Finucane, H.K., Bulik-Sullivan, B., Gusev, A., Trynka, G., Reshef, Y., Loh, P.R., Anttila, V., Xu, H., Zang, C., Farh, K., et al. (2015). Partitioning heritability by functional annotation using genome-wide association summary statistics. Nat Genet 47, 1228-1235.

25. Huber, C.D., DeGiorgio, M., Hellmann, I., and Nielsen, R. (2016). Detecting recent selective sweeps while controlling for mutation rate and background selection. Mol Ecol 25, 142156.

26. Vitti, J.J., Grossman, S.R., and Sabeti, P.C. (2013). Detecting natural selection in genomic data. Annu Rev Genet 47, 97-120.

27. Lek, M., Karczewski, K.J., Minikel, E.V., Samocha, K.E., Banks, E., Fennell, T., O'DonnellLuria, A.H., Ware, J.S., Hill, A.J., Cummings, B.B., et al. (2016). Analysis of proteincoding genetic variation in 60,706 humans. Nature 536, 285-291.

28. McVicker, G., Gordon, D., Davis, C., and Green, P. (2009). Widespread genomic signatures of natural selection in hominid evolution. PLoS Genet 5, e1000471.

29. Sabeti, P.C., Varilly, P., Fry, B., Lohmueller, J., Hostetter, E., Cotsapas, C., Xie, X., Byrne, E.H., McCarroll, S.A., Gaudet, R., et al. (2007). Genome-wide detection and characterization of positive selection in human populations. Nature 449, 913-918. 
30. Grossman, S.R., Andersen, K.G., Shlyakhter, I., Tabrizi, S., Winnicki, S., Yen, A., Park, D.J., Griesemer, D., Karlsson, E.K., Wong, S.H., et al. (2013). Identifying recent adaptations in large-scale genomic data. Cell 152, 703-713.

31. Grossman, S.R., Shlyakhter, I., Karlsson, E.K., Byrne, E.H., Morales, S., Frieden, G., Hostetter, E., Angelino, E., Garber, M., Zuk, O., et al. (2010). A composite of multiple signals distinguishes causal variants in regions of positive selection. Science 327, 883886.

32. Durvasula, A., and Sankararaman, S. (2019). A statistical model for reference-free inference of archaic local ancestry. PLoS Genet 15, e1008175.

33. Sankararaman, S., Mallick, S., Dannemann, M., Prufer, K., Kelso, J., Paabo, S., Patterson, N., and Reich, D. (2014). The genomic landscape of Neanderthal ancestry in present-day humans. Nature 507, 354-357.

34. Dall'Olio, G.M., Bertranpetit, J., Wagner, A., and Laayouni, H. (2014). Human genome variation and the concept of genotype networks. PLoS One 9, e99424.

35. Dall'Olio, G.M., Vahdati, A.R., Bertranpetit, J., Wagner, A., and Laayouni, H. (2015). VCF2Networks: applying genotype networks to single-nucleotide variants data. Bioinformatics 31, 438-439.

36. Wagner, A. (2008). Neutralism and selectionism: a network-based reconciliation. Nat Rev Genet 9, 965-974.

37. Altshuler, D.M., Gibbs, R.A., Peltonen, L., Altshuler, D.M., Gibbs, R.A., Peltonen, L., Dermitzakis, E., Schaffner, S.F., Yu, F., Peltonen, L., et al. (2010). Integrating common and rare genetic variation in diverse human populations. Nature 467, 52-58. 
38. Yu, D., Sul, J.H., Tsetsos, F., Nawaz, M.S., Huang, A.Y., Zelaya, I., Illmann, C., Osiecki, L., Darrow, S.M., Hirschtritt, M.E., et al. (2019). Interrogating the Genetic Determinants of Tourette's Syndrome and Other Tic Disorders Through Genome-Wide Association Studies. Am J Psychiatry 176, 217-227.

39. Wood, S.N., Pya, N., and Säfken, B. (2016). Smoothing Parameter and Model Selection for General Smooth Models. Journal of the American Statistical Association 111, 1548-1563. 40. Benjamini, Y., and Yekutieli, D. (2001). The Control of the False Discovery Rate in Multiple Testing under Dependency. The Annals of Statistics 29, 1165-1188.

41. Dannemann, M., and Kelso, J. (2017). The Contribution of Neanderthals to Phenotypic Variation in Modern Humans. Am J Hum Genet 101, 578-589.

42. McCoy, R.C., Wakefield, J., and Akey, J.M. (2017). Impacts of Neanderthal-Introgressed Sequences on the Landscape of Human Gene Expression. Cell 168, 916-927 e912.

43. Demontis, D., Walters, R.K., Martin, J., Mattheisen, M., Als, T.D., Agerbo, E., Baldursson, G., Belliveau, R., Bybjerg-Grauholm, J., Baekvad-Hansen, M., et al. (2019). Discovery of the first genome-wide significant risk loci for attention deficit/hyperactivity disorder. Nat Genet 51, 63-75.

44. Watson, H.J., Yilmaz, Z., Thornton, L.M., Hubel, C., Coleman, J.R.I., Gaspar, H.A., Bryois, J., Hinney, A., Leppa, V.M., Mattheisen, M., et al. (2019). Genome-wide association study identifies eight risk loci and implicates metabo-psychiatric origins for anorexia nervosa. Nat Genet 51, 1207-1214.

45. Grove, J., Ripke, S., Als, T.D., Mattheisen, M., Walters, R.K., Won, H., Pallesen, J., Agerbo, E., Andreassen, O.A., Anney, R., et al. (2019). Identification of common genetic risk variants for autism spectrum disorder. Nat Genet 51, 431-444. 
46. Stahl, E.A., Breen, G., Forstner, A.J., McQuillin, A., Ripke, S., Trubetskoy, V., Mattheisen, M., Wang, Y., Coleman, J.R.I., Gaspar, H.A., et al. (2019). Genome-wide association study identifies 30 loci associated with bipolar disorder. Nat Genet 51, 793-803.

47. Howard, D.M., Adams, M.J., Clarke, T.K., Hafferty, J.D., Gibson, J., Shirali, M., Coleman, J.R.I., Hagenaars, S.P., Ward, J., Wigmore, E.M., et al. (2019). Genome-wide metaanalysis of depression identifies 102 independent variants and highlights the importance of the prefrontal brain regions. Nat Neurosci 22, 343-352.

48. Schizophrenia Working Group of the Psychiatric Genomics Consortium (2014) Biological insights from 108 schizophrenia-associated genetic loci. Nature 511, 421-427.

49. Levey, D.F., Gelernter, J., Polimanti, R., Zhou, H., Cheng, Z., Aslan, M., Quaden, R., Concato, J., Radhakrishnan, K., Bryois, J., et al. (2020). Reproducible Genetic Risk Loci for Anxiety: Results From approximately 200,000 Participants in the Million Veteran Program. Am J Psychiatry, appiajp201919030256.

50. Polimanti, R., and Gelernter, J. (2017). Widespread signatures of positive selection in common risk alleles associated to autism spectrum disorder. PLoS Genet 13, e1006618.

51. Hujoel, M.L.A., Gazal, S., Hormozdiari, F., van de Geijn, B., and Price, A.L. (2019). Disease Heritability Enrichment of Regulatory Elements Is Concentrated in Elements with Ancient Sequence Age and Conserved Function across Species. Am J Hum Genet 104, 611-624.

52. Charlesworth, B. (2012). The effects of deleterious mutations on evolution at linked sites. Genetics 190, 5-22. 
53. Comeron, J.M., Williford, A., and Kliman, R.M. (2008). The Hill-Robertson effect: evolutionary consequences of weak selection and linkage in finite populations. Heredity (Edinb) 100, 19-31.

54. North, T.L., and Beaumont, M.A. (2015). Complex trait architecture: the pleiotropic model revisited. Sci Rep 5, 9351.

55. Stein, M.B., Levey, D.F., Cheng, Z., Wendt, F.R., Harrington, K., Cho, K., Quaden, R., Radhakrishnan, K., Girgenti, M.J., Anne Ho, Y.-L., et al. (2019). Genomic Characterization of Posttraumatic Stress Disorder in a Large US Military Veteran Sample. bioRxiv, 764001.

56. Ivleva, E.I., Moates, A.F., Hamm, J.P., Bernstein, I.H., O'Neill, H.B., Cole, D., Clementz, B.A., Thaker, G.K., and Tamminga, C.A. (2014). Smooth pursuit eye movement, prepulse inhibition, and auditory paired stimuli processing endophenotypes across the schizophrenia-bipolar disorder psychosis dimension. Schizophr Bull 40, 642-652.

57. Kikuchi, M., Miura, K., Morita, K., Yamamori, H., Fujimoto, M., Ikeda, M., Yasuda, Y., Nakaya, A., and Hashimoto, R. (2018). Genome-wide Association Analysis of Eye Movement Dysfunction in Schizophrenia. Sci Rep 8, 12347.

58. Obyedkov, I., Skuhareuskaya, M., Skugarevsky, O., Obyedkov, V., Buslauski, P., Skuhareuskaya, T., and Waszkiewicz, N. (2019). Saccadic eye movements in different dimensions of schizophrenia and in clinical high-risk state for psychosis. BMC Psychiatry 19, 110.

59. Byrne, R.P., van Rheenen, W., van den Berg, L.H., Veldink, J.H., and McLaughlin, R.L. (2020). Dutch population structure across space, time and GWAS design. bioRxiv, 2020.2001.2001.892513. 
bioRxiv preprint doi: https://doi.org/10.1101/2020.02.26.966531; this version posted February 27, 2020. The copyright holder for this preprint

(which was not certified by peer review) is the author/funder. All rights reserved. No reuse allowed without permission.

\section{Figure titles and legends}

\section{Figure 1 Enrichment of natural selection and functional annotation measures. Significant}

enrichments of genic and loss-of-function (LoF) intolerant loci (A) and three genomic

annotations of background selection (BGS). Both panels list phenotypes in descending order

from highest to lowest magnitude enrichment for the most abundant annotation (i.e., LoF

intolerance in panel A and top 2\% of BGS scores in panel B). All phenotypes listed are at least

nominally significant $(p<0.05)$ and solid circles indicate that the enrichment survived multiple

testing correction $(q<0.05)$. Error bars represent the $95 \%$ confidence interval around each

enrichment estimate.
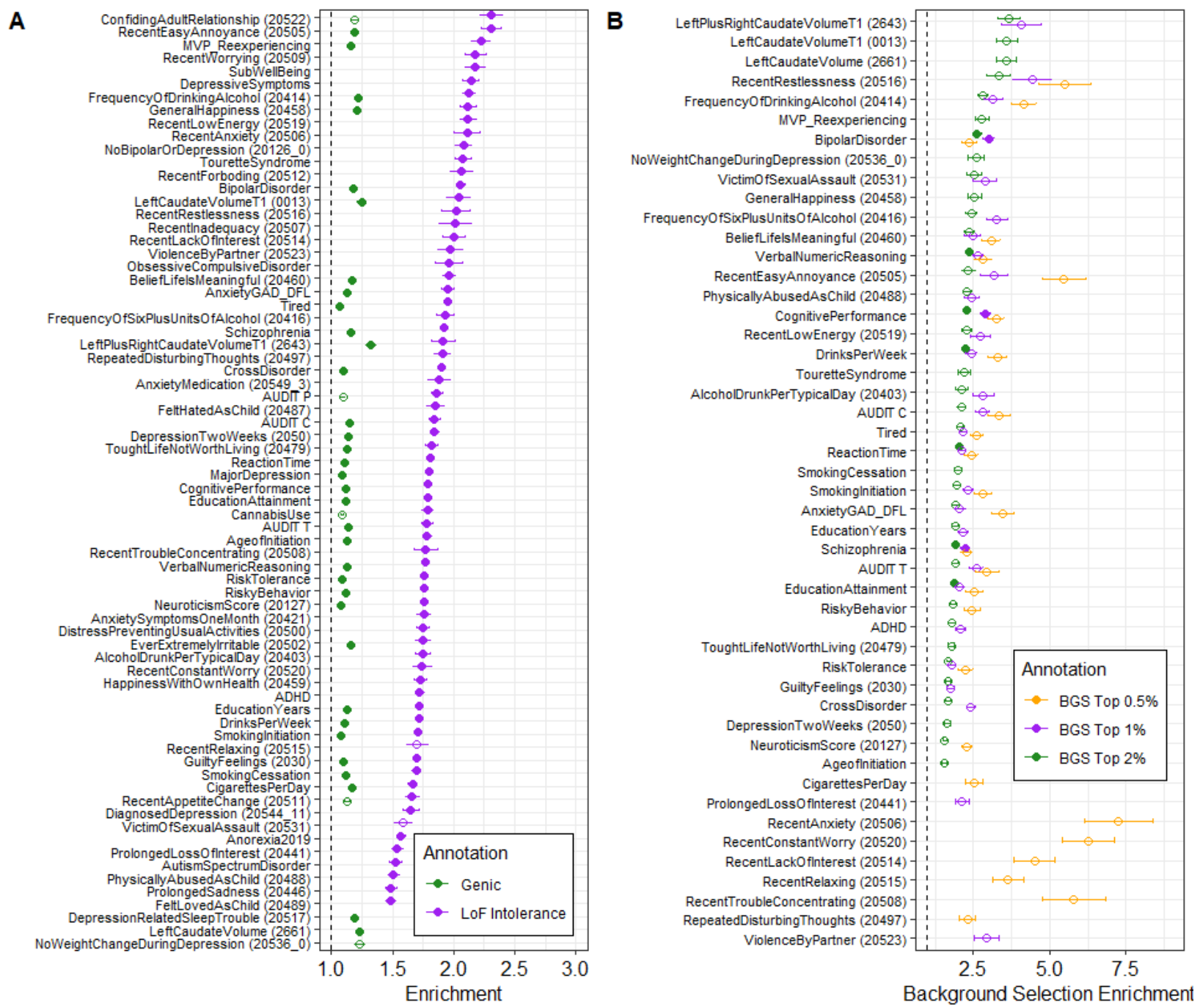
Figure 2 Complex trait effect size distribution curves. Effect size distribution curves derived from GWAS summary statistics for 75 mental health and disease phenotypes using the GENESIS R package. Phenotypes are grouped into eleven categories (Table S1). The phenotype corresponding to the steepest curve $(\uparrow)$ and the widest curve $(\leftrightarrow)$ are identified in each subplot.
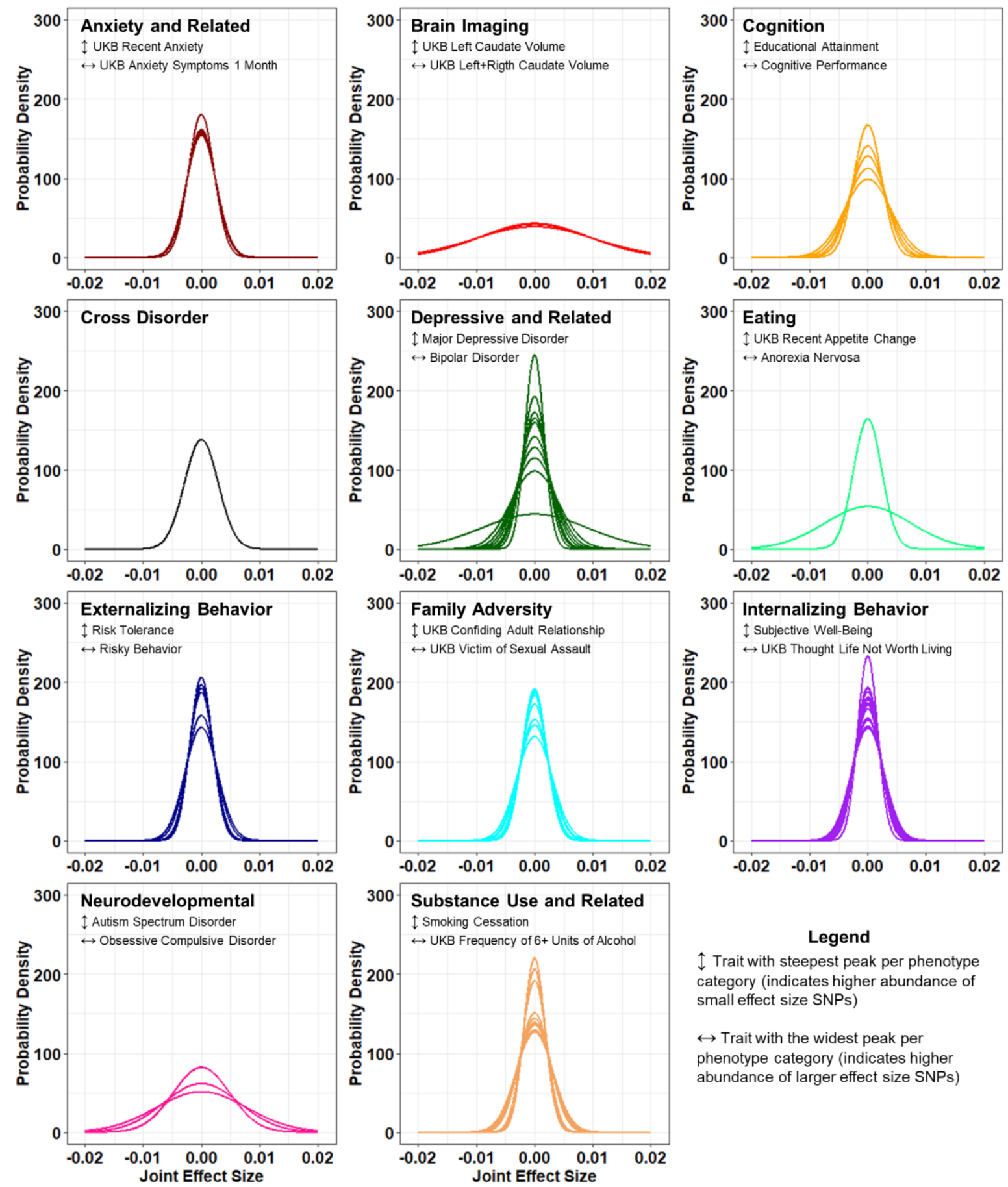

Legend

$\uparrow$ Trait with steepest peak per phenotype category (indicates higher abundance of small effect size SNPs)

$\leftrightarrow$ Trait with the widest peak per phenotype category (indicates higher abundance of larger effect size SNPs) 
Figure 3 Correlation between effect size distribution, natural selection, and functional annotation. Spearman correlation between effect size distribution descriptive statistics and heritability $\left(h^{2}\right)$, enrichment for background selection (BGS) partitions (top 2\%, 1\%, and 0.5\%), genic loci, and loss-of-function intolerant (LoF Intol.) loci. Panel A correlates z-scores for all measures, panel B correlates the $z$-score for all measures against unstandardized effect size distribution descriptive statistics, panel C correlates unstandardized enrichment and $h^{2}$ magnitudes against unstandardized effect size distribution descriptive statistics. Large text indicates significant correlation after multiple testing correction $(q<0.05)$.
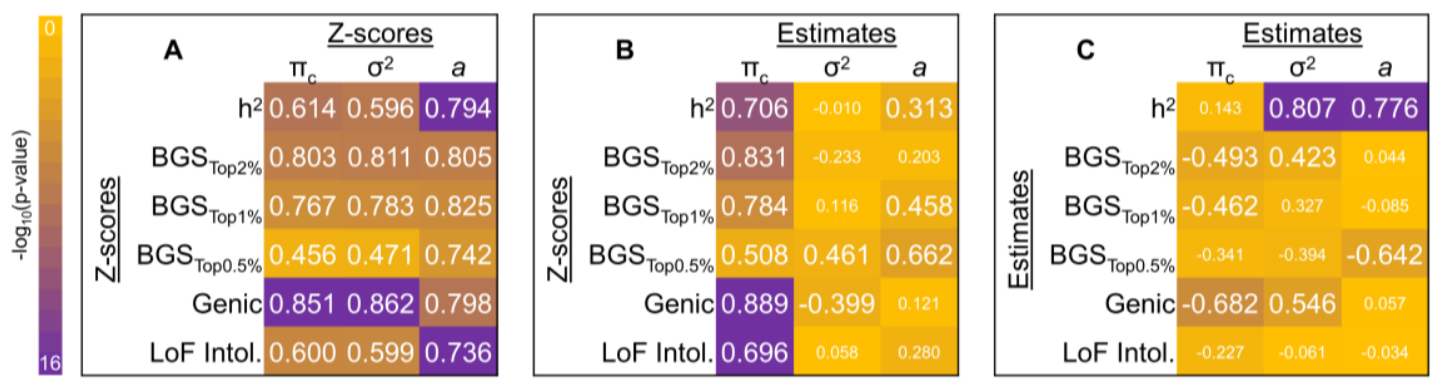


\section{Figure 4 Predicting effect size distribution with natural selection, functional annotation,}

and phenotype heterogeneity. Summary of models predicting effect size distribution

parameters. (A) Median-based linear models (MBLM) for predicting effect size distribution

descriptive statistics with a single unstandardized independent variable. Larger font indicates at least nominal significance for the respective effect of each independent variable on the outcome effect size distribution descriptive statistic. (B and C) Generalized (GAM) additive (B) and interactive (C) models of effect size distribution descriptive statistics using individually significant predictors of each metric (from A). Prior to testing the addition of heterogeneity information, each model was re-evaluated in psychiatric disorders only.

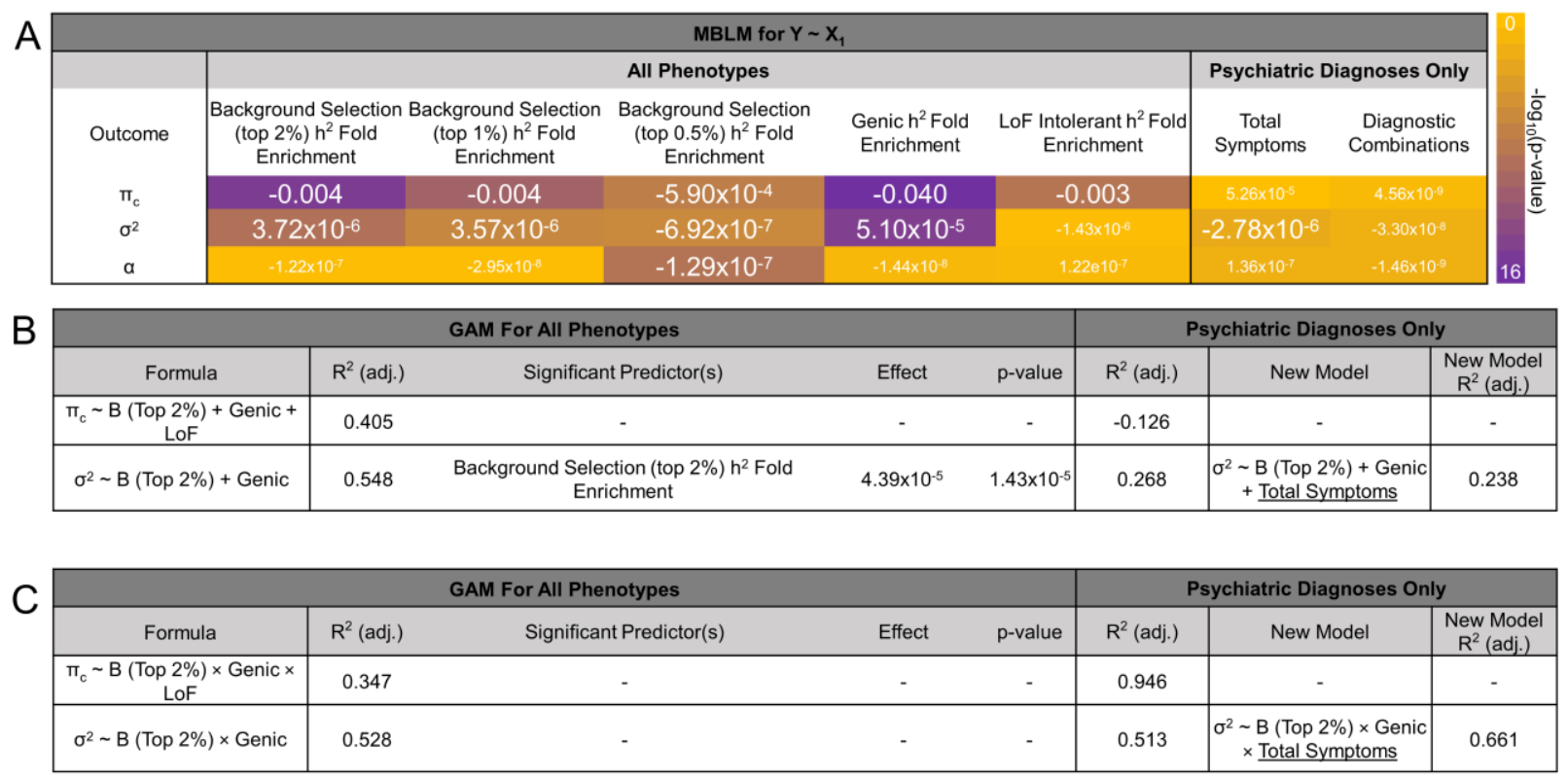




\section{Tables}

Table 1 Functional annotation enrichments. Three phenotypes whose observed scale heritability $\left(h^{2}\right)$ estimates demonstrated significant $(q<0.05)$ enrichment of loci in genic and lossof-function $(\mathrm{LoF})$ intolerant regions of the genome.

\begin{tabular}{|l|c|c|}
\hline \multicolumn{1}{|c|}{ Trait } & Genic $^{2}$ Fold Enrichment $(p)$ & LoF Intolerance ${ }^{2}$ Fold Enrichment $(p)$ \\
\hline Schizophrenia & $1.16\left(5.46 \times 10^{-11}\right)$ & $1.92\left(8.80 \times 10^{-21}\right)$ \\
\hline Educational Attainment & $1.12\left(1.70 \times 10^{-10}\right)$ & $1.79\left(8.65 \times 10^{-23}\right)$ \\
\hline Cognitive Performance & $1.12\left(2.71 \times 10^{-9}\right)$ & $1.79\left(6.26 \times 10^{-29}\right)$ \\
\hline
\end{tabular}

Table 2 Background selection enrichments. Seven phenotypes whose observed scale heritability $\left(h^{2}\right)$ estimates demonstrated significant enrichment $(q<0.05)$ of background selection (BGS) in at least one genomic annotation of per-SNP $B$-statistic measures. FDR significant observations $(q<0.05)$ are in bold text.

\begin{tabular}{|c|c|c|c|}
\hline Trait & $\begin{array}{c}\text { BGS }(\text { top } 2 \%) h^{2} \text { Fold } \\
\text { Enrichment }(p)\end{array}$ & $\begin{array}{l}\text { BGS (top 1\%) } h^{2} \text { Fold } \\
\text { Enrichment }(p)\end{array}$ & $\begin{array}{c}\text { BGS (top 0.5\%) } h^{2} \text { Fold } \\
\text { Enrichment }(p)\end{array}$ \\
\hline Bipolar Disorder & $2.62\left(2.01 \times 10^{-6}\right)$ & $3.01\left(4.31 \times 10^{-6}\right)$ & $2.36(0.021)$ \\
\hline Cognitive Performance & $2.29\left(1.81 \times 10^{-8}\right)$ & $2.90\left(7.31 \times 10^{-6}\right)$ & $3.24(0.001)$ \\
\hline Drinks Per Week & $2.25\left(8.33 \times 10^{-7}\right)$ & $2.43(0.004)$ & $3.29(0.006)$ \\
\hline Educational Attainment & $1.86\left(9.69 \times 10^{-6}\right)$ & $2.04(0.001)$ & $2.53(0.003)$ \\
\hline Schizophrenia & $1.90\left(7.29 \times 10^{-6}\right)$ & $2.23\left(6.04 \times 10^{-6}\right)$ & $2.27(0.007)$ \\
\hline Reaction Time & $2.02\left(1.33 \times 10^{-7}\right)$ & $2.13(0.001)$ & $2.44(0.018)$ \\
\hline Verbal Numeric Reasoning & $2.35\left(8.63 \times 10^{-8}\right)$ & $2.63\left(2.21 \times 10^{-4}\right)$ & $2.81(0.017)$ \\
\hline
\end{tabular}




\section{Supplementary Information}

Table S1. Description of all phenotypes, data sources, and statistics derived from each GWAS of the mental health and disease phenome. Nominally significant enrichments are provided; bolded enrichments survive multiple testing correction $(q<0.05)$ [xlsx file attached]. 
Figure S1. Robust linear (orange) and local (LOESS, purple) relationships between heritability ( $h^{2}$ estimate and $z$-score) and enrichment of $h^{2}$ attributed to genic and loss-of-function (LoF) intolerant SNPs (estimate and z-score) for phenotypes demonstrating nominally significant enrichment of these annotations. The $h^{2}$ of labeled phenotypes was FDR significantly $(q<0.05$, Table 1) enriched for both genic and LoF intolerant SNPs. Note that robust linear regression down-weighs data points by their distance from the regression line resulting in data point distributions visually inconsistent with a linear model (e.g., middle and right panels in the bottom row).
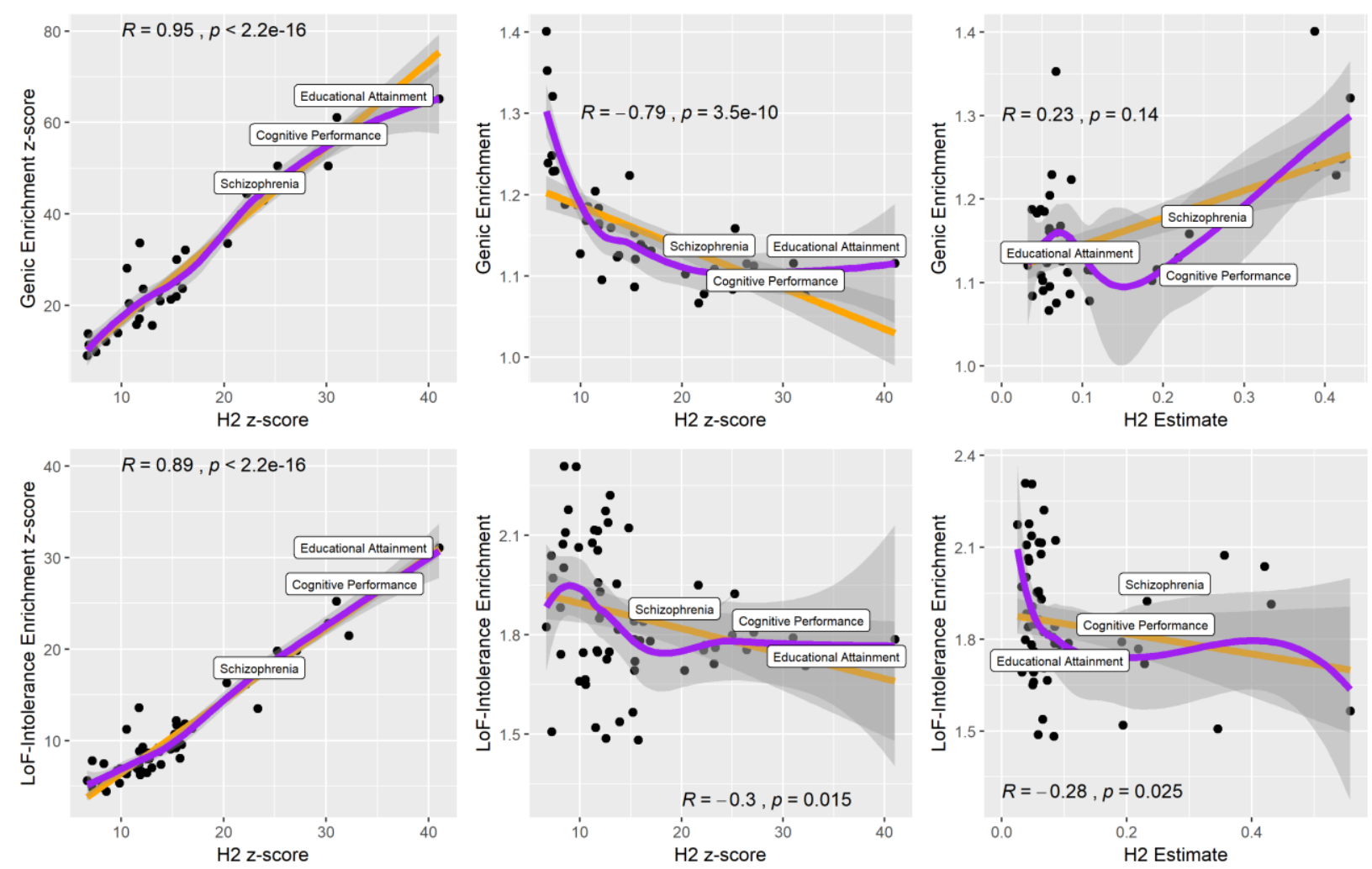
Figure S2. Robust linear (orange) and local (LOESS, purple) relationships between heritability ( $h^{2}$ estimate and $z$-score) and enrichment of $h^{2}$ attributed to background selection (BGS) intolerant SNPs (estimate and z-score) for phenotypes demonstrating nominally significant enrichment of these annotations. The $h^{2}$ of labeled phenotypes was FDR significantly $(q<0.05$, Table 2$)$ enriched for both genic and LoF intolerant SNPs.
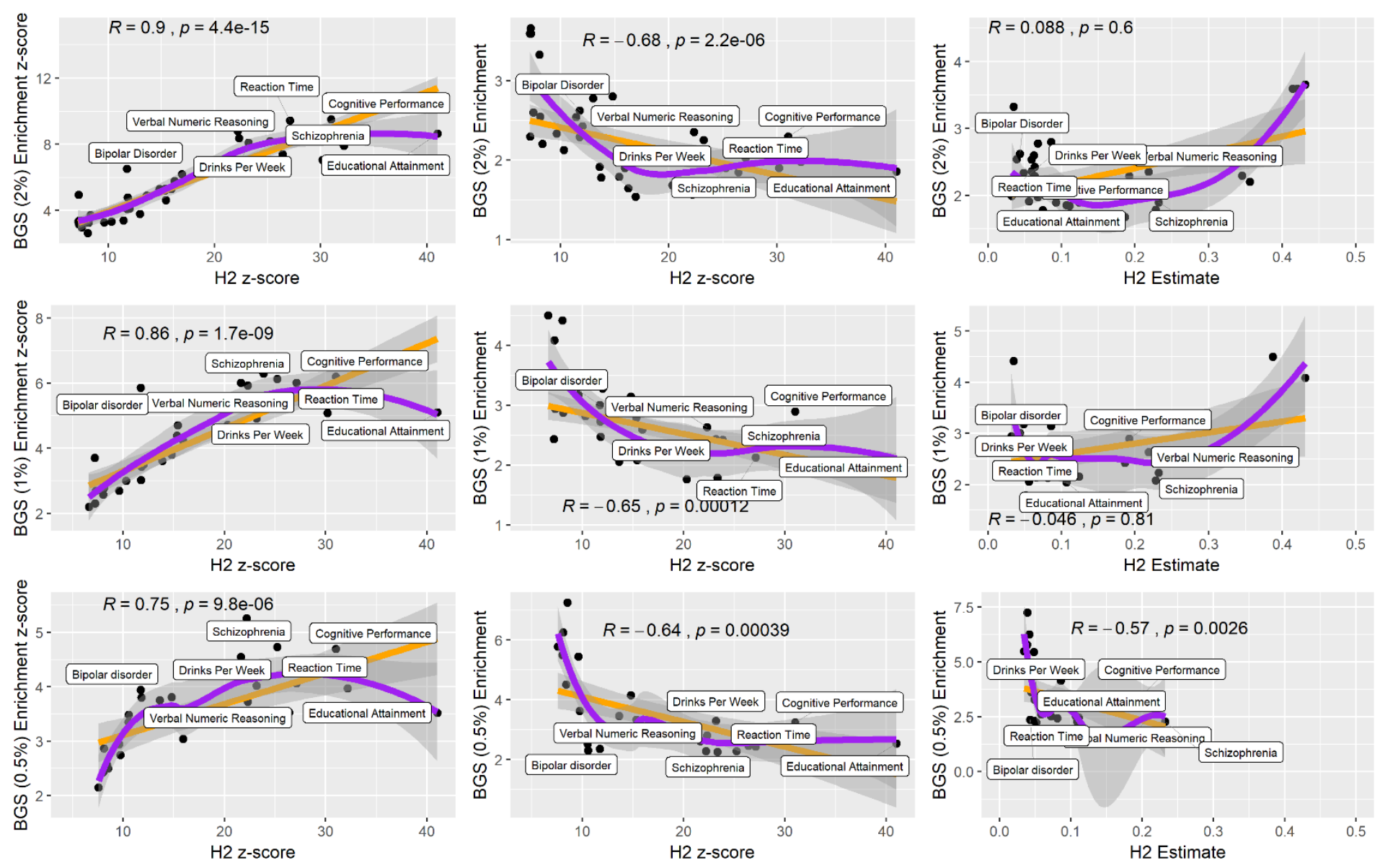